\title{
Effect of Atrial Fibrillation Duration on Probability of Immediate Recurrence after Transthoracic Cardioversion
}

\author{
HAKAN ORAL, M.D., MEHMET OZAYDIN, M.D., CHRISTIAN STICHERLING, M.D., \\ HIROSHI TADA, M.D., CHRISTOPH SCHARF, M.D., AMAN CHUGH, M.D., \\ STEVE W.K. LAI, M.D., FRANK PELOSI JR., M.D., BRADLEY P. KNIGHT, M.D., \\ S. ADAM STRICKBERGER, M.D., and FRED MORADY, M.D.
}

From the Division of Cardiology, Department of Internal Medicine, University of Michigan, Ann Arbor, Michigan, USA

\begin{abstract}
Immediate Recurrence of Atrial Fibrillation. Introduction: An immediate recurrence of atrial fibrillation (IRAF) appears to be more common after early restoration of sinus rhythm with an implantable atrial defibrillator than after elective transthoracic cardioversion, which suggests that the probability of IRAF may be related to the duration of AF.

Methods and Results: Transthoracic cardioversion was performed $85 \pm 187$ days (range 7 minutes to 8 years) after the onset of atrial fibrillation in 315 patients (mean age $61 \pm 13$ years). IRAF was defined as a recurrence of $\mathrm{AF}$ within 60 seconds after restoration of sinus rhythm. IRAF occurred in $56 \%$ of patients when cardioversion was performed within 1 hour of the onset of $A F$ compared with $12 \%$ of patients when cardioversion was performed after 24 hours of $\mathrm{AF}(\mathrm{P}<0.001)$. The duration of AF was the only independent predictor of IRAF among the clinical variables of age, gender, structural heart disease, antiarrhythmic drug therapy, and cardioversion energy $(P<0.01)$.

Conclusion: IRAF is more likely to occur when the duration of AF is $<1$ hour than when the duration is $>\mathbf{2 4}$ hours. This observation has clinical implications for the most appropriate timing of cardioversion, particularly in patients who receive device therapy for AF. (J Cardiovasc Electrophysiol, Vol. 14, pp. 182-185, February 2003)
\end{abstract}

atrial fibrillation, cardioversion, immediate recurrence of atrial fibrillation, pulmonary vein

\section{Introduction}

The prevalence of an immediate recurrence of atrial fibrillation (IRAF) after transthoracic cardioversion has been reported to range from $5 \%$ to $26 \% .{ }^{1-4}$ However, IRAF appears to be more common after internal or epicardial cardioversion; it has been reported to occur in $27 \%$ to $50 \%$ of patients after these types of cardioversion. ${ }^{5-7}$ The reason why IRAF may be more likely to occur after internal or epicardial cardioversion than after transthoracic cardioversion is unclear. One difference among these types of cardioversion is that, compared with transthoracic cardioversion, cardioversion with an implanted defibrillator or epicardial electrodes generally is performed sooner after the onset of atrial fibrillation. The aim of this prospective study was to determine the effect of the duration of atrial fibrillation on the probability of IRAF after transthoracic cardioversion.

\section{Methods}

\section{Patient Characteristics}

The subjects of this prospective study were 315 patients ( 256 men and 59 women, mean age $61 \pm 13$ years) who under-

Supported in part by the Ellen and Robert Thompson Atrial Fibrillation Research Fund.

Address for correspondence: Hakan Oral, M.D., Division of Cardiology, TC B1D226, University of Michigan, 1500 E. Medical Center Drive, Ann Arbor, MI 48109-0311. Fax: 734-936-7026; E-mail: oralh@umich.edu

Manuscript received 17 September 2002; Accepted for publication 27 November 2002. went transthoracic cardioversion of atrial fibrillation. Atrial fibrillation had been present for a mean duration of $85 \pm$ 187 days (range 7 minutes to 8 years). Structural heart disease was present in $146(46 \%)$ of the 315 patients: 77 (24\%) had coronary artery disease, $34(11 \%)$ had valvular heart disease, $34(11 \%)$ had a nonischemic cardiomyopathy, and 1 had an atrial septal defect. Among the 315 patients, 29 (9\%) were being treated with a Class I antiarrhythmic drug and $89(28 \%)$ with a Class III drug. Among the 315 subjects, 308 (98\%) had a history of clinical atrial fibrillation.

\section{Study Protocol}

All patients provided written informed consent. Among the 315 patients, cardioversion was performed during a catheter ablation procedure in 92 patients (29\%) who had either spontaneous or pacing-induced atrial fibrillation during the procedure. Overall, the episode of atrial fibrillation that was cardioverted in this study was spontaneous in 273 (87\%) of the 315 patients and pacing-induced in 42 (13\%).

The duration of atrial fibrillation was determined based on the clinical history and analysis of all available ECG recordings. Patients in whom the duration of atrial fibrillation could not be accurately determined were not included in the study.

IRAF was defined as a recurrence of atrial fibrillation within 60 seconds after restoration of sinus rhythm by transthoracic cardioversion. A continuous ECG recording was performed during and for at least 2 minutes after cardioversion.

\section{Transthoracic Cardioversion}

If atrial fibrillation had been present for $>48$ hours, cardioversion was performed either after at least 3 weeks of 
anticoagulation with warfarin and an INR $>2$ or after a transesophageal echocardiogram that did not show any intracardiac thrombi and systemic anticoagulation with heparin. Conscious sedation or anesthesia was achieved with midazolam, fentanyl, and/or propafol. Transthoracic cardioversion was performed in 267 patients (85\%) with monophasic shocks (Hewlett-Packard, Andover, MA, USA) and in 48 patients (15\%) with biphasic shocks (Zoll Inc., Burlington, MA, USA). Adhesive electrodes were positioned on the anterior and posterior chest. Monophasic shock strengths were 200 to $360 \mathrm{~J}$, and biphasic shock strengths were 120 to $200 \mathrm{~J}$. When cardioversion was performed during a catheter ablation procedure, caution was exercised to avoid contact of any of the catheters against the myocardium to prevent catheter-induced depolarizations during cardioversion.

When atrial fibrillation had been present for $>48$ hours, therapeutic anticoagulation was continued for at least 6 weeks after cardioversion. Antiarrhythmic therapy was continued at the discretion of the treating physicians.

\section{Statistical Analysis}

Continuous variables are expressed as mean \pm 1 SD. Continuous variables were compared with Student's $t$-test. Categorical variables were compared by Chi-square analysis or with the Fisher exact test, as appropriate. Multivariate logistic regression analysis was performed to determine the independent predictors of IRAF. $\mathrm{P}<0.05$ was considered statistically significant.

\section{Results}

\section{Duration of Atrial Fibrillation}

The mean duration of atrial fibrillation was $85 \pm 187$ days (range 7 minutes to 8 years). Among the 315 patients, the duration of atrial fibrillation was $<1$ hour in $48(15 \%), 1$ to 24 hours in $27(9 \%), 1$ day to 1 week in $34(11 \%), 1$ to 4 weeks in $45(14 \%), 1$ to 3 months in $72(23 \%), 3$ to 6 months in $36(11 \%), 6$ to 12 months in $40(13 \%)$, and 1 to 8 years in $13(4 \%)$.

\section{Overall Prevalence of IRAF}

IRAF occurred in 63 (20\%) of 315 patients who underwent cardioversion for an episode of atrial fibrillation. The mean time to IRAF was $20 \pm 25$ seconds after cardioversion.

\section{Duration of Atrial Fibrillation and IRAF}

The relationship between the duration of atrial fibrillation and the prevalence of IRAF is shown in Figure 1. The prevalence of IRAF was $56 \%$ when the duration of atrial fibrillation was $<1$ hour, $37 \%$ when the duration was 1 to 24 hours, and $12 \%$ when the duration was $>24$ hours $(\mathrm{P}<0.001)$. Beyond a duration of 1 day, there was no relationship between the duration of atrial fibrillation and the prevalence of IRAF.

\section{Shock Waveform and Strength}

IRAF occurred in $52(21 \%)$ of 267 patients when sinus rhythm was restored with a monophasic shock and in $8(17 \%)$ of 48 patients who were cardioverted with a biphasic shock $(\mathrm{P}=0.5)$.

The mean shock strength that restored sinus rhythm was similar in patients who had IRAF and in those who did not

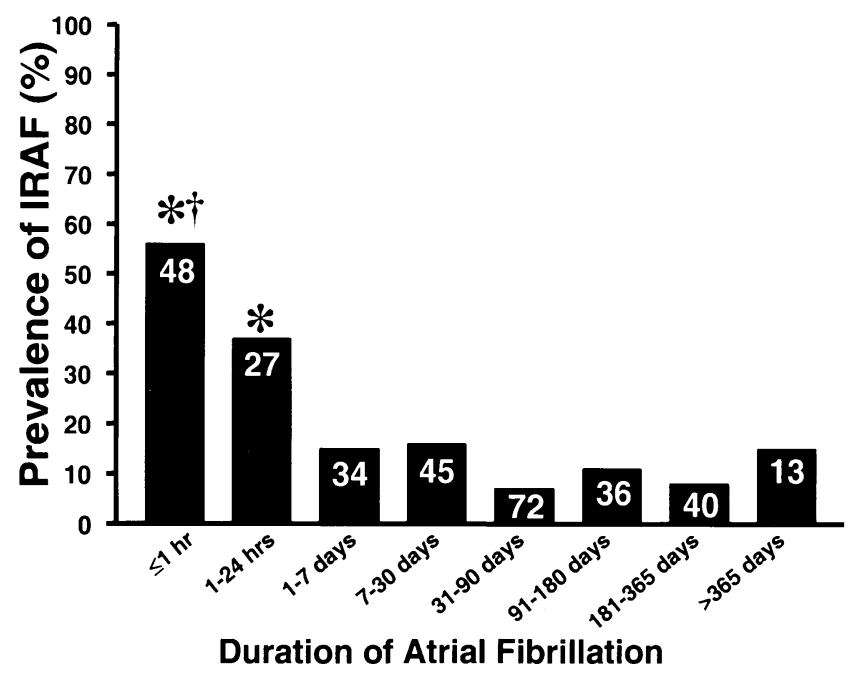

Figure 1. Prevalence of immediate recurrence of atrial fibrillation (IRAF) and duration of atrial fibrillation. Patients were categorized into eight groups based on the duration of atrial fibrillation before cardioversion. The number of patients in each category is shown in white numbers within each bar. ${ }^{*} P<$ 0.05 vs patients with atrial fibrillation of $>24$ hours; $\dagger P<0.05$ vs patients with atrial fibrillation $>1$ hour and $\leq 1$ day.

have IRAF (monophasic: $200 \pm 96 \mathrm{~J}$ vs $220 \pm 68 \mathrm{~J}, \mathrm{P}=0.2$; biphasic $129 \pm 49 \mathrm{~J}$ vs $135 \pm 43 \mathrm{~J} ; \mathrm{P}=0.7$ ).

\section{Multivariate Predictors of IRAF}

Among the variables of age, gender, duration of atrial fibrillation, presence of structural heart disease, concomitant antiarrhythmic drug therapy, and cardioversion energy, only the duration of atrial fibrillation was an independent predictor of IRAF $(\mathrm{P}<0.01)$.

\section{Duration of Atrial Fibrillation and IRAF During a Catheter Ablation Procedure}

Among the subgroup of patients in whom cardioversion was performed during a catheter ablation procedure, IRAF occurred in $37(57 \%)$ of 65 patients when the duration of atrial fibrillation was $<24$ hours and in $9(33 \%)$ of 27 patients when the duration was $>24$ hours $(\mathrm{P}<0.04)$.

\section{IRAF after Cardioversion of Spontaneous and Pacing-Induced Atrial Fibrillation}

To determine whether the prevalence of IRAF was similar after cardioversion of spontaneous and induced episodes of atrial fibrillation, the prevalence of IRAF was compared among patients who underwent cardioversion during a catheter ablation procedure and had either spontaneous or pacing-induced atrial fibrillation for $<24$ hours. IRAF occurred in $9(69 \%)$ of 13 patients after cardioversion of an episode of spontaneous atrial fibrillation and in $28(56 \%)$ of 50 patients after cardioversion of an episode of atrial fibrillation that was induced by pacing $(\mathrm{P}=0.4)$.

\section{Discussion}

\section{Main Findings}

The main finding of this study is that there is an inverse relationship between the duration of atrial fibrillation and the 
prevalence of IRAF after transthoracic cardioversion. The prevalence of IRAF was high $(56 \%)$ when atrial fibrillation had been present for $<1$ hour, intermediate $(37 \%)$ when it had been present for 1 to 24 hours, and relatively low (12\%) when it had been present for $>24$ hours. Furthermore, among several clinical variables examined, the duration of atrial fibrillation was the only independent predictor of IRAF after cardioversion.

\section{Possible Mechanisms of IRAF}

There are several possible factors that may contribute to recurrences of atrial fibrillation immediately after cardioversion, including changes in autonomic tone, electrophysiologic changes in the atrial substrate, ${ }^{8}$ and premature depolarizations that trigger atrial fibrillation. ${ }^{1}$ However, in a prior study, IRAF was consistently abolished by electrical isolation of the pulmonary veins, providing strong evidence that the most important determinant of IRAF is the ectopy that arises from the muscle sleeves that surround the pulmonary veins. ${ }^{9}$

Given that the primary determinant of IRAF may be arrhythmogenic foci in the pulmonary veins, the results of the present study suggest that these arrhythmogenic foci may be most active when atrial fibrillation has been present for $<1$ hour and that the foci become progressively less active as the duration of atrial fibrillation lengthens.

The cellular mechanisms of pulmonary vein arrhythmogenicity remain largely unclear. Although triggered activity may be a mechanism of arrhythmias arising in the pulmonary veins, abnormal automaticity and reentry cannot be excluded ${ }^{10-12}$ Regardless of the cellular mechanisms, it has been demonstrated that the generation of these arrhythmias depends on input from the left atrium. ${ }^{11}$ It is possible that arrhythmogenic pulmonary venous foci are activated by the rapid atrial activity that occurs during atrial fibrillation but that as the duration of atrial fibrillation lengthens, the cellular mechanisms responsible for this arrhythmogenic activity are progressively down-regulated or deactivated. Although the present study demonstrates that the probability of IRAF is inversely related to the duration of atrial fibrillation, it provides no insights into mechanisms, and the explanation for this observation remains to be determined.

\section{Immediate versus Early Recurrence of Atrial Fibrillation}

Several studies have clearly demonstrated that the probability of recurrent atrial fibrillation after cardioversion is directly related to the duration of atrial fibrillation ${ }^{13-16}$ and that recurrences are most likely to occur within the first few days or weeks after cardioversion. ${ }^{2}$ Therefore, it is important to distinguish these early recurrences of atrial fibrillation from the phenomenon of IRAF, which occurs within 1 minute after cardioversion. Although the probability of early recurrences of atrial fibrillation increases as the duration of atrial fibrillation increases, the opposite is true for recurrences of atrial fibrillation that occur immediately after cardioversion. This strongly suggests that the mechanisms that underlie early and immediate recurrences of atrial fibrillation are different. It is possible that arrhythmogenic foci within the pulmonary veins are responsible for IRAF, ${ }^{9}$ whereas progressive electrical and anatomic remodeling are responsible for early recurrences of atrial fibrillation. ${ }^{17,18}$

\section{Previous Studies}

When attempting to localize the sites of origin of the triggers for atrial fibrillation, it is useful to induce atrial fibrillation by rapid pacing and then restore sinus rhythm shortly afterward by electrical cardioversion. This clinical practice is useful because it frequently evokes IRAF, allowing localization of the trigger sites. ${ }^{19}$ The results of the present study explain why IRAF is commonly evoked in the electrophysiology laboratory, given that cardioversion typically is performed within minutes after induction of atrial fibrillation.

A recent retrospective study in patients with an implantable atrial defibrillator demonstrated that a duration of atrial fibrillation of $<3$ hours is a predictor of recurrence within few minutes after cardioversion. ${ }^{7}$ However, in this prior study, any atrial arrhythmia was categorized as atrial fibrillation, and the prevalence of immediate recurrences of atrial fibrillation probably was underestimated because of limitations in the detection algorithms of the implantable devices. For example, successful cardioversion followed 1 or 2 seconds later by atrial fibrillation likely would be categorized as unsuccessful cardioversion instead of IRAF. Based on the results of the present study, it is clear that the heightened predisposition to IRAF after internal cardioversion is not related to any direct effect of shocks delivered within the heart but simply to the short duration of atrial fibrillation prior to cardioversion. This also applies to the high prevalence of IRAF when epicardial electrodes are used for cardioversion of atrial fibrillation in patients who have undergone open heart surgery. ${ }^{6}$

\section{Study Limitations}

A limitation of this study is that there are two possible confounding variables that may have affected the probability of IRAF. The first was the presence of catheters in the heart in patients who underwent cardioversion of short-lived atrial fibrillation in the electrophysiology laboratory. Despite attempts to position the catheters away from the atrial wall, it is possible that mechanical irritation of the atrium by these catheters accounted for some episodes of IRAF. The second possible confounding variable was the method by which atrial fibrillation was provoked. Atrial fibrillation always was spontaneous in onset when elective cardioversion was performed outside of the electrophysiology laboratory, but it often was induced by rapid pacing when cardioversion was performed for short-lived episodes of atrial fibrillation in the electrophysiology laboratory. However, subgroup analysis of patients who underwent cardioversion in the electrophysiology laboratory demonstrated that the prevalence of IRAF still was higher in patients with atrial fibrillation for $<24$ hours than in patients with atrial fibrillation for $>24$ hours. Moreover, the prevalence of IRAF was similar regardless of whether atrial fibrillation was spontaneous or induced by atrial pacing. These findings suggest that intracardiac catheters and the method of atrial fibrillation induction were not significant confounding variables.

\section{Clinical Implications}

The concept that "atrial fibrillation begets atrial fibrillation" 17 has encouraged early cardioversion of atrial fibrillation to avoid the progressive electrical and structural remodeling that occurs during atrial fibrillation. However, the results 
of the present study suggest that there is a lower limit to the ideal timing of cardioversion and that a more satisfactory clinical outcome possibly can be achieved if cardioversion is postponed for approximately 24 hours. Because the probability of IRAF is greatest when sinus rhythm is restored within 1 hour after the onset of atrial fibrillation and remains high for 24 hours, device therapies designed to restore sinus rhythm shortly after the onset of atrial fibrillation or treatment algorithms that utilize very early cardioversion may have a high probability of evoking IRAF. The ideal timing for cardioversion may be at approximately 24 hours, when the probability of IRAF has significantly diminished and before electrical remodeling has become pronounced. Additional prospective studies are necessary to establish the ideal timing for elective cardioversion of atrial fibrillation.

\section{References}

1. Daoud EG, Hummel JD, Augostini R, Williams S, Kalbfleisch SJ: Effect of verapamil on immediate recurrence of atrial fibrillation. J Cardiovasc Electrophysiol 2000;11:1231-1237.

2. Tieleman RG, Van Gelder IC, Crijns HJ, De Kam PJ, Van Den Berg MP, Haaksma J, Van Der Woude HJ, Allessie MA: Early recurrences of atrial fibrillation after electrical cardioversion: A result of fibrillation-induced electrical remodeling of the atria? J Am Coll Cardiol 1998;31:167-173.

3. Van Noord T, Van Gelder IC, Schoonderwoerd BA, Crijns HJ: Immediate reinitiation of atrial fibrillation after electrical cardioversion predicts subsequent pharmacologic and electrical conversion to sinus rhythm and amiodarone. Am J Cardiol 2000;86:1384-1385.

4. Crijns HJ, van Noord T, van Gelder IC: Recurrence of atrial fibrillation and the need for new definitions. Eur Heart J 2001;22:1769-1771.

5. Wellens HJ, Lau CP, Luderitz B, Akhtar M, Waldo AL, Camm AJ, Timmermans C, Tse HF, Jung W, Jordaens L, Ayers G: Atrioverter: An implantable device for the treatment of atrial fibrillation. Circulation 1998;98:1651-1656.

6. Liebold A, Wahba A, Birnbaum DE: Low-energy cardioversion with epicardial wire electrodes: New treatment of atrial fibrillation after open heart surgery. Circulation 1998;98:883-886.

7. Schwartzman D, Musley SK, Swerdlow C, Hoyt RH, Warman EN: Early recurrence of atrial fibrillation after ambulatory shock conversion. J Am Coll Cardiol 2002;40:93-99.
8. Duytschaever M, Danse P, Allessie M: Supervulnerable phase immediately after termination of atrial fibrillation. J Cardiovasc Electrophysiol 2002:13:267-275

9. Oral H, Knight BP, Ozaydin M, Chugh A, Hassan S, Scharf C, Lai SW, Greenstein R, Han J, Pelosi F, Strickberger SA, Morady F: Segmental ostial ablation to isolate the pulmonary veins during atrial fibrillation: Feasibility and mechanistic insights. Circulation 2002;106:1256-1262.

10. Chen SA, Hsieh MH, Tai CT, Tsai CF, Prakash VS, Yu WC, Hsu TL, Ding YA, Chang MS: Initiation of atrial fibrillation by ectopic beats originating from the pulmonary veins: Electrophysiological characteristics, pharmacological responses, and effects of radiofrequency ablation. Circulation 1999;100:1879-1886.

11. Oral H, Ozaydin M, Tada H, Chugh A, Scharf C, Hassan S, Lai S, Greenstein R, Pelosi F Jr, Knight BP, Strickberger SA, Morady F: Mechanistic significance of intermittent pulmonary vein tachycardia in patients with atrial fibrillation. J Cardiovasc Electrophysiol 2002;13:645-650.

12. Oral H, Knight BP, Tada H, Morady F: Tachycardia and bradycardia coexisting in the same pulmonary vein. J Cardiovasc Electrophysiol 2002;13:186-188.

13. Dittrich HC, Erickson JS, Schneiderman T, Blacky AR, Savides T, Nicod PH: Echocardiographic and clinical predictors for outcome of elective cardioversion of atrial fibrillation. Am J Cardiol 1989;63:193197.

14. Van Gelder IC, Crijns HJ, Van Gilst WH, Verwer R, Lie KI: Prediction of uneventful cardioversion and maintenance of sinus rhythm from directcurrent electrical cardioversion of chronic atrial fibrillation and flutter. Am J Cardiol 1991;68:41-46.

15. Van Gelder IC, Crijns HJ, Tieleman RG, Brugemann J, De Kam PJ, Gosselink AT, Verheugt FW, Lie KI: Chronic atrial fibrillation. Success of serial cardioversion therapy and safety of oral anticoagulation. Arch Intern Med 1996;156:2585-2592.

16. Levy S, Ricard P, Lau CP, Lok NS, Camm AJ, Murgatroyd FD, Jordaens LJ, Kappenberger LJ, Brugada P, Ripley KL: Multicenter low energy transvenous atrial defibrillation (XAD) trial results in different subsets of atrial fibrillation. J Am Coll Cardiol 1997;29:750-755

17. Wijffels MC, Kirchhof CJ, Dorland R, Allessie MA: Atrial fibrillation begets atrial fibrillation. A study in awake chronically instrumented goats. Circulation 1995;92:1954-1968.

18. Kim BS, Kim YH, Hwang GS, Pak HN, Lee SC, Shim WJ, Oh DJ, Ro YM: Action potential duration restitution kinetics in human atrial fibrillation. J Am Coll Cardiol 2002;39:1329-1336.

19. Lau CP, Tse HF, Ayers GM: Defibrillation-guided radiofrequency ablation of atrial fibrillation secondary to an atrial focus. J Am Coll Cardiol 1999;33:1217-1226. 